\title{
Efficacy and predictive biomarkers of immunotherapy in Epstein-Barr virus- associated gastric cancer
}

\author{
Yuezong Bai, ${ }^{1}$ Tong Xie, ${ }^{1}$ Zhenghang Wang, ${ }^{1}$ Shuang Tong, ${ }^{2}$ Xiaochen Zhao, ${ }^{2}$ \\ Feilong Zhao, ${ }^{2}$ Jinping Cai, ${ }^{2}$ Xiaofan Wei, ${ }^{3}$ Zhi Peng, ${ }^{1}$ Lin Shen (i) ${ }^{1}$
}

To cite: Bai Y, Xie T, Wang Z, et al. Efficacy and predictive biomarkers of immunotherapy in Epstein-Barr virus-associated gastric cancer. Journal for ImmunoTherapy of Cancer 2022;10:e004080. doi:10.1136/ jitc-2021-004080

- Additional supplemental material is published online only. To view, please visit the journal online (http://dx.doi.org/10. 1136/jitc-2021-004080).

YB and TX contributed equally.

Accepted 31 January 2022

A Check for updates

(c) Author(s) (or their employer(s)) 2022. Re-use permitted under CC BY-NC. No commercial re-use. See rights and permissions. Published by BMJ.

${ }^{1}$ Department of Gastrointestinal Oncology, Key Laboratory of Carcinogenesis and Translational Research (Ministry of Education), Peking University Cancer Hospital \& Institute, Beijing, China

${ }^{2}$ Medical Affairs, 3D Medicines, Inc, Shanghai, China

${ }^{3}$ Department of Human Anatomy, Histology and Embryology,

School of Basic Medical

Sciences, Peking University

Health Science Center, Beijing,

China

Correspondence to

Professor Lin Shen;

linshenpku@163.com

Dr Zhi Peng;

zhipeng3@hotmail.com

\section{ABSTRACT}

Background Epstein-Barr virus (EBV)-associated gastric cancer (GC) (EBVaGC) is a distinct molecular subtype of $\mathrm{GC}$ with a favorable prognosis. However, the exact effects and potential mechanisms of EBV infection on immune checkpoint blockade (ICB) efficacy in GC remain to be clarified. Additionally, EBV-encoded RNA (EBER) in situ hybridization (ISH), the traditional method to detect EBV, could cause false-positive/false-negative results and not allow for characterizing other molecular biomarkers recommended by standard treatment guidelines for GC. Herein, we sought to investigate the efficacy and potential biomarkers of ICB in EBVaGC identified by next-generation sequencing (NGS).

Design An NGS-based algorithm for detecting EBV was established and validated using two independent GC cohorts (124 in the training cohort and 76 in the validation cohort). The value of EBV infection for predicting ICB efficacy was evaluated among 95 patients with advanced or metastatic $\mathrm{GC}$ receiving ICB. The molecular predictive biomarkers for ICB efficacy were identified to improve the prediction accuracy of ICB efficacy in 22 patients with EBVaGC.

Results Compared with orthogonal assay (EBER-ISH) results, the NGS-based algorithm achieved high performance with a sensitivity of $95.7 \%(22 / 23)$ and a specificity of $100 \%(53 / 53)$. EBV status was identified as an independent predictive factor for overall survival and progression-free survival in patients with DNA mismatch repair proficient (pMMR) GC following ICB. Moreover, the patients with EBV+/pMMR and EBV_/ MMR deficient (dMMR) had comparable and favorable survival following ICB. Twenty-two patients with EBV+/pMMR achieved an objective response rate of $54.5 \%$ (12/22) on immunotherapy. Patients with EBVaGC with a high cytotoxic T lymphocyteassociated antigen-4 (CTLA-4) level were less responsive to anti-programmed death-1/igand 1 (PD-1/L1) monotherapy, and the combination of anti-CTLA-4 plus anti-PD-1/L1 checkpoint blockade benefited patients with EBVaGC more than anti-PD-1/ L1 monotherapy with a trend close to significance $(p=0.074)$. There were nearly significant differences in tumor mutational burden (TMB) level and SMARCA4 mutation frequency between the ICB response and non-response group.

Conclusions We developed an efficient NGS-based EBV detection strategy, and this strategy-identified EBV infection was as effective as dMMR in predicting ICB efficacy in GC. Additionally, we identified CTLA-4, TMB, and SMARCA4 mutation as potential predictive biomarkers of ICB efficacy in EBVaGC, which might better inform ICB treatment for EBVaGC.

\section{BACKGROUND}

Epstein-Barr virus (EBV)-associated gastric cancer (GC) (EBVaGC) accounts for approximately $5 \%-10 \%$ of GC worldwide and is well recognized as a distinct molecular subtype of GC. ${ }^{1-4}$ Several lines of evidence suggested that patients with EBVaGC tended to have fewer lymph node metastases and a better prognosis. $^{5-7}$ Among cohorts of advanced EBVaGC, response rates following antiprogrammed death-1 (anti-PD-1) monoimmunotherapy were reported to range from $25 \%$ to $100 \%$, but all higher than that of unselected patients with advanced GC. ${ }^{8-11}$ Although the exact impacts and potential mechanisms of EBV infection on GC immune checkpoint blockade (ICB) efficacy remain to be clarified, there has been a growing interest in EBV as an emerging biomarker to inform clinical management of GC, especially ICB treatment.

EBV-encoded RNA (EBER) in situ hybridization (ISH) has long been regarded as the gold standard for detecting EBV. However, apart from commonly false-negative EBER-ISH results caused by RNA degradation, false-positive results might be generated in the presence of background hybridization caused by poorly fixed tissues, non-specific staining, or cross-reactivity. ${ }^{12} 13$ Moreover, EBER-ISH does not allow for simultaneous characterization of other clinically relevant biomarkers such as tumor mutational burden (TMB), microsatellite instability (MSI), HER2 amplification, and NTRK fusion as recommended by the National Comprehensive Cancer Network (NCGN) Guidelines for GC. ${ }^{14}$ The advent of next-generation sequencing (NGS) offers a viable solution by accommodating EBV detection and the gene profile in numerous other cancerrelated markers in one single assay. However, it has been only employed to interrogate the EBV genome for research purposes. ${ }^{15} 16$ The 
development and validation of NGS-based EBV detection intended for clinical use have never been reported.

In this work, we aimed to develop an efficient NGSbased EBV detection strategy that allows for parallel characterization of other genomic features. More importantly, we assessed the ability of EBV status to predict the benefit from ICB treatment and explored the predictive molecular markers which may be incorporated into EBV status to improve the accuracy of ICB efficacy prediction.

\section{METHODS}

\section{Samples and study design}

The study design and consort patient flow diagram were illustrated in online supplemental figures 1 and 2. Gene selection, algorithm development, and EBV score cutoff training were performed using 24 EBVaGC and 100 EBV-negative GC (EBVnGC) tissue samples. Technical validation was conducted in a cohort of $23 \mathrm{EBVaGC}$ and 53 EBVnGC tissue samples. The 124 and 76 samples mentioned above were obtained from the Beijing Cancer Hospital with a confirmed histological diagnosis of advanced GC and available EBER-ISH results.

The correlation between EBV score and viral copy number by quantitative PCR (qPCR) was assessed using 20 GC DNA samples with EBV score $>0.00005$ (excluding EBV genes that could not be detected at all) retrieved from the 3DMed Biobank (3D Medicines).

The repeatability and reproducibility of the EBV detection method were evaluated using four EBVaGC and four EBVnGC tissue samples randomly selected from the technical validation cohort. Each sample was detected for two runs with each run in quadruplicate under the same operating conditions. The data of replicates in the intraassay were counted for the repeatability test, while the data of the two batches in the inter-assay precision study were collected and compared for reproducibility analysis.

To determine the limit of detection (LOD), EBVtransformed B lymphoblasts, BL1954, BL1395, BL2009, and BL1143, were purchased from the American Type Culture Collection, and the control white blood cells were obtained from a healthy donor. ${ }^{1718}$ Briefly, genomic DNA samples derived from four EBV-positive cell lines were diluted into the genomic DNA isolated from the control white blood cells, targeting four titration points, $2.5 \%$, $5 \%, 10 \%$, and $20 \%$. The titration series were examined at a total cell input of $5 \times 10^{6}$ and four different sequencing depths: $100 \times, 300 \times, 500 \times$, and $1000 \times$.

To evaluate the predictive value of EBV status in ICB efficacy in patients with GC and further identify the molecular markers predictive of ICB efficacy in patients with EBVaGC, 95 patients with advanced or metastatic GC treated with ICB at Beijing Cancer Hospital from June 21, 2017, to October 22, 2021, were included. The exact ICB drugs for these patients were summarized in online supplemental table 1 . Among 95 patients, 66 were DNA mismatch repair (MMR) proficient (pMMR), and 29 were MMR deficient (dMMR), where MMR status was identified by immunohistochemistry (IHC). The patients with pMMR comprised 22 patients with EBV+/ pMMR and 44 patients with EBV-/pMMR, wherein EBV status was obtained via our NGS-based method. The patients with dMMR were EBV negative and represented as EBV-/dMMR phenotype. All tumor samples had at least $20 \%$ tumor content as reviewed by two independent pathologists.

IHC, qPCR, NGS, EBER-ISH, and multiplex immunofluorescence $(\mathrm{mIF})$ were described in online supplemental methods and online supplemental table 6 .

\section{ICB efficacy evaluation}

De-identified clinicopathological and efficacy data were extracted from patients' medical records by two independent physicians and were reviewed by a third physician in case of inconsistency. Tumor response was assessed as per the Response Evaluation Criteria in Solid Tumors, V.1.1, and categorized as complete response (CR), partial response (PR), stable disease (SD), and disease progression (PD). Objective response rate (ORR) was defined as CR plus PR. Progression-free survival (PFS) was defined as the time from the onset of ICB treatment to PD or death, whichever occurred first. Overall survival (OS) was defined as the time from the onset of ICB treatment to death as a result of any cause. The duration of response was defined as the interval from first documented CR or PR until PD or death by any cause, whichever occurred first. All samples were obtained with informed consents.

\section{Statistical analyses}

Continuous variables were compared using a Student's t-test or the non-parametric Mann-Whitney $\mathrm{U}$ test, while categorical variables were compared using the $\chi^{2}$ test or the Fisher's exact test where appropriate. Survival curves were plotted with the Kaplan-Meier method and analyzed using a log-rank test. Univariate and multivariate Cox regression analyses were applied to identify independent prognostic variables for ICB efficiency. Simple linear regression was adopted to examine the relationship between EBV copy number and EBV score. The receiver operating characteristic (ROC) analysis was performed using the web tool EasyROC (http://www.biosoft. hacettepe.edu.tr/easyROC/). All tests were two-sided, and a $p$ value of $<0.05$ was considered statistically significant. Statistical analyses were performed using R software V.3.6.1 (R Foundation for Statistical Computing), Python software V.3.9.5, GraphPad Prism V.7.01 (GraphPad Software), and SPSS V.22.0 (IBM).

\section{RESULTS}

\section{Algorithm development for EBV detection}

The study design was illustrated in online supplemental figure 1. To select target genes for EBV detection, $B H R F 1$ and $B C L F 1$ were excluded upfront for sharing significant homology with the human genome. ${ }^{19}{ }^{20}$ Regions with a high GC content were also excluded due to affecting library preparation/construction. ${ }^{21}$ The rest of the 
genome sequences were evaluated by their relevance to EBV pathogenesis and carcinogenesis, and six genes, EBNA-1, EBNA-2, EBNA-3, LMP1, LMP2, and BZLF1, were included in the EBV detection panel. ${ }^{22}{ }^{23}$ For each gene, a set of probes was designed to cover the whole exons. Since EBV viruses are classified into type 1 and type 2 based on EBNA-2 and EBNA-3 sequences, two sets of probes targeting type 1 and type 2 sequences separately were developed for each of the two genes.

Tissue samples from 24 patients with EBVaGC and 100 patients with EBVnGC as a training set were subjected to NGS analysis using a panel combining the EBV detection probes and the probes covering the whole exons of 733 cancer-related genes, including all currently available biomarkers related to tumor immunotherapy, targeted therapy, chemoradiotherapy, and prognosis (the gene list shown in online supplemental table 2). The sequencing depth was calculated for each EBV gene and multiplied by two before normalization using the sequencing depth of the 733-panel genes to obtain a normalized depth (NorDepth) for each gene. Due to low capture efficiency, LMP1 and LMP2 were excluded (online supplemental figure 3). The remaining four genes had a sharply higher NorDepth in the tumors from patients with EBVaGC than EBVnGC (figure 1A), which were included in the final panel. EBV score was defined as the median of the NorDepths of these four genes. In the training set, EBV score could significantly discriminate EBVaGC from EBVnGC ( $<<0.001)$ (figure 1B).

Additionally, 20 GC DNA samples were quantified for EBV load by qPCR. A strong correlation was observed between EBV score and EBV copy number $\left(R^{2}=0.9326\right.$, $\mathrm{p}<0.001$, figure $1 \mathrm{C}$ ), corroborating the reliability of EBV score to reflect EBV status. The optimal cut-off EBV score for the definition of EBV positivity was determined at 0.05695 using the ROC curves, with an area under the curve (AUC) of 1. Furthermore, we found that the positive relation between EBV score and EBV copy number also held for the individual EBV gene, and the AUC values for EBV positivity predictions were 0.969 for $E B N A$ 1, 0.865 for EBNA-2, 0.875 for EBNA-3, and 0.906 for $B Z L F 1$ (online supplemental figure 4A,B). These results indicated that the EBV algorithm based on NGS detection of four EBV genes was established and could accurately identify EBVaGC.

\section{Technical validation}

The accuracy of the EBV algorithm defining EBVaGC with an EBV score of at least 0.05695 was validated in a cohort of 76 advanced GC tumor samples, where 23 were diagnosed as EBVaGC and 53 as EBVnGC by EBER-ISH previously. Our NGS-based method identified 95.7\% (22/23, 95\% CI $77.3 \%$ to $99.8 \%$ ) of EBVaGC tumors and $100 \%$ (53/53, 95\% CI $93.2 \%$ to $100 \%)$ of the EBVnGC tumors, for an overall accuracy of $98.7 \%$ (75/76, 95\% CI $92.9 \%$ to $99.9 \%$ ) (figure 1D, online supplemental table 3 ). The positive predictive value was $100 \%$ (22/22, 95\% CI $85.1 \%$ to $100 \%)$. The repeatability and reproducibility of the
EBV detection method were also assessed. Eight samples tested in two batches could get the same EBV status with $100 \%$ concordance (online supplemental table 4 ). The results of LOD showed that for all four EBV-positive cell lines, the EBV score of each sample was well above 0.05695 when the dilution was above $5 \%$ across different sequencing depths (figure 1E). Therefore, the LOD was determined as $5 \%$ at a sequencing depth of $100 \times$.

\section{EBV infection predicts clinical benefit from ICB}

As existing evidence on the sensitivity of patients with EBVaGC to ICB remained controversial, ${ }^{8-10} 24$ 95 patients with advanced or metastatic GC receiving ICB therapy were included to evaluate the predictive value of EBV infection in ICB efficacy. In 95 patients, 29 were dMMR, and 66 were pMMR consisting of 22 patients with $\mathrm{EBV}+/$ pMMR and 44 patients with EBV-/pMMR. EBV status in patients with pMMR was identified by our NGS-based method. Patients' baseline characteristics were summarized in table 1 . In patients with pMMR, patients with $\mathrm{EBV}+/ \mathrm{pMMR}$ had a significantly higher proportion of responders than patients with EBV-/pMMR ( $p=0.008$ ) (figure 2A). The survival analyses showed that patients with $\mathrm{EBV}+/ \mathrm{pMMR}$ had significantly favorable PFS (median PFS (mPFS) 8.5 vs 2.0 months, $\mathrm{p}<0.001$ ) and OS (median OS (mOS) not reached (NR) vs 5.0 months, $\mathrm{p}=0.002$ ) after ICB compared with patients with EBV-/pMMR (figure 2B,C). Univariate survival analyses revealed that ICB strategy, EBV status, prior systemic therapy, and age were significantly associated with PFS and OS (figure 2D, (online supplemental table 5). Multivariate analysis indicated that EBV status remained a strong prognostic factor for PFS (HR 0.39, 95\% CI 0.16 to $0.97, \mathrm{p}=0.042$ ) in patients with pMMR GC following ICB (figure 2E). Of note, there were two patients with EBV-negative by NGS and EBV-positive by EBER-ISH, both of whom were categorized into the EBV-/pMMR set. In the two patients, the PFS was 2.0 and 3.0 months, respectively, and OS was 3.2 and 10.4 months, respectively, which verged on the mPFS (2.0 months) and mOS (5.0 months) of the EBV-/ pMMR group.

MSI-H/dMMR is a well-established biomarker for immunotherapy. ${ }^{14}$ In the 22 patients with EBV+/pMMR, that is, EBVaGC, 12 achieved PR, five showed SD, and five experienced PD, yielding an ORR of $54.5 \%$ (95\% CI $33.7 \%$ to $75.4 \%$ ) (figure $2 \mathrm{~F}$ ), which was significantly higher than the ORR of $17.7 \%(\mathrm{p}=0.008)$ in the EBV-/ pMMR group and comparable to the EBV-/dMMR group ( $\mathrm{p}=0.768$, online supplemental figure $5 \mathrm{~A}, \mathrm{~B}$ ) independent from the ICB strategy (data not shown). The median time to response was 1.8 months (range 1.1-5.6 months) in patients with EBVaGC (figure 2G). Besides, the ORR to ICB in patients with EBVaGC was less affected by prior lines of therapy $(\mathrm{p}=0.378)$ (online supplemental figure 6 ). The survival analyses showed that the patients with $\mathrm{EBV}+/ \mathrm{pMMR}$ and EBV-/dMMR had comparable and favorable PFS and OS, both of whom derived more survival benefit from ICB than the patients with EBV-/ 
A

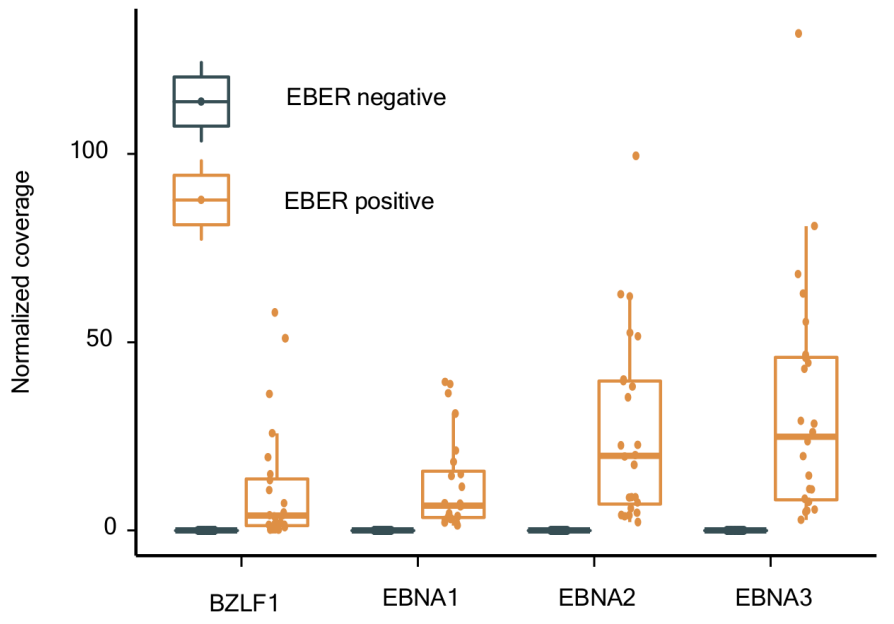

C

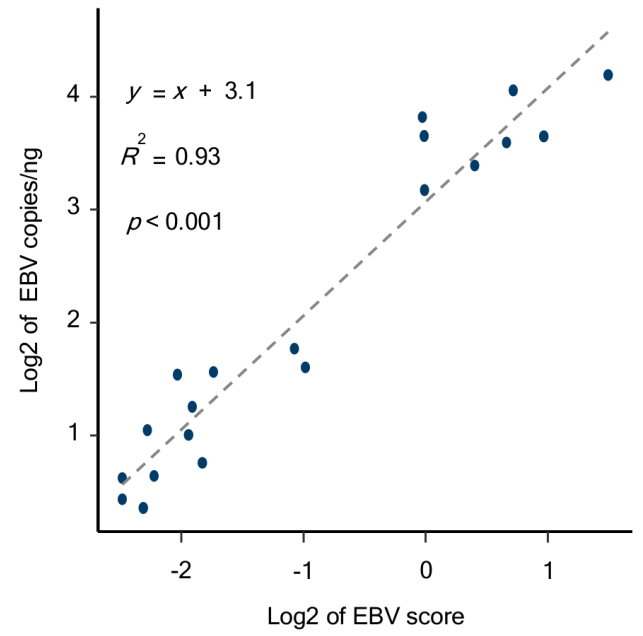

B

D
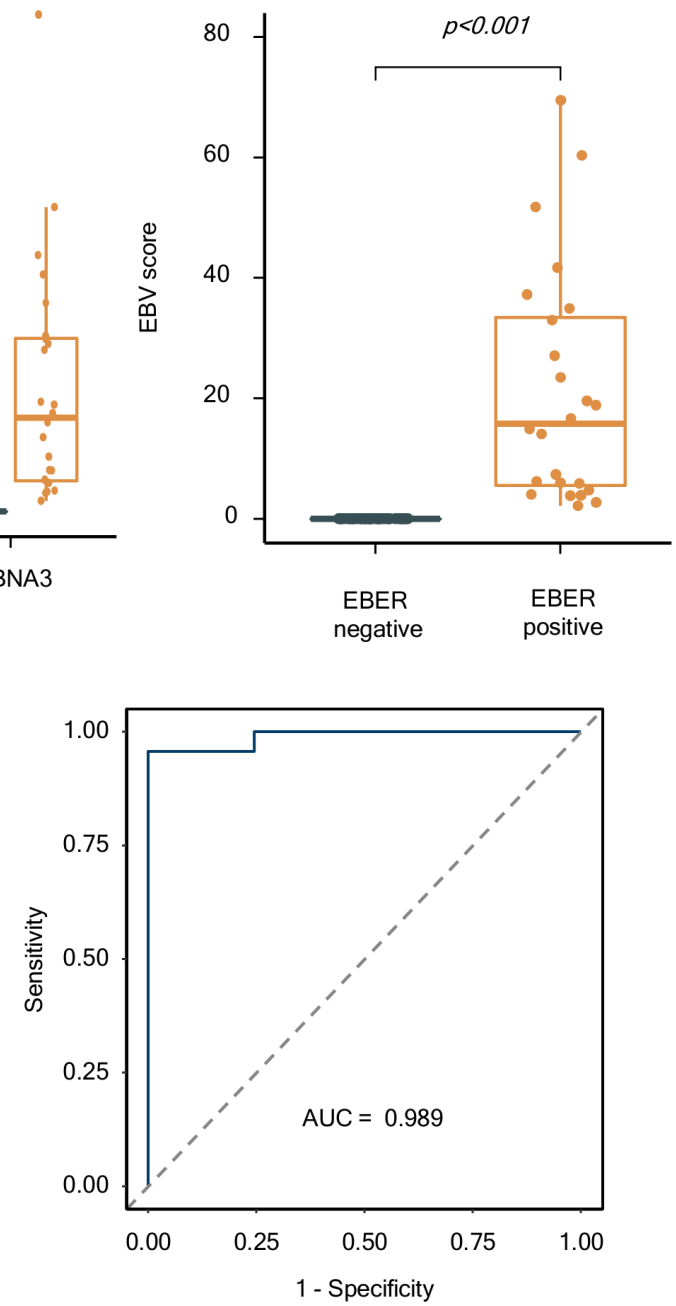

E

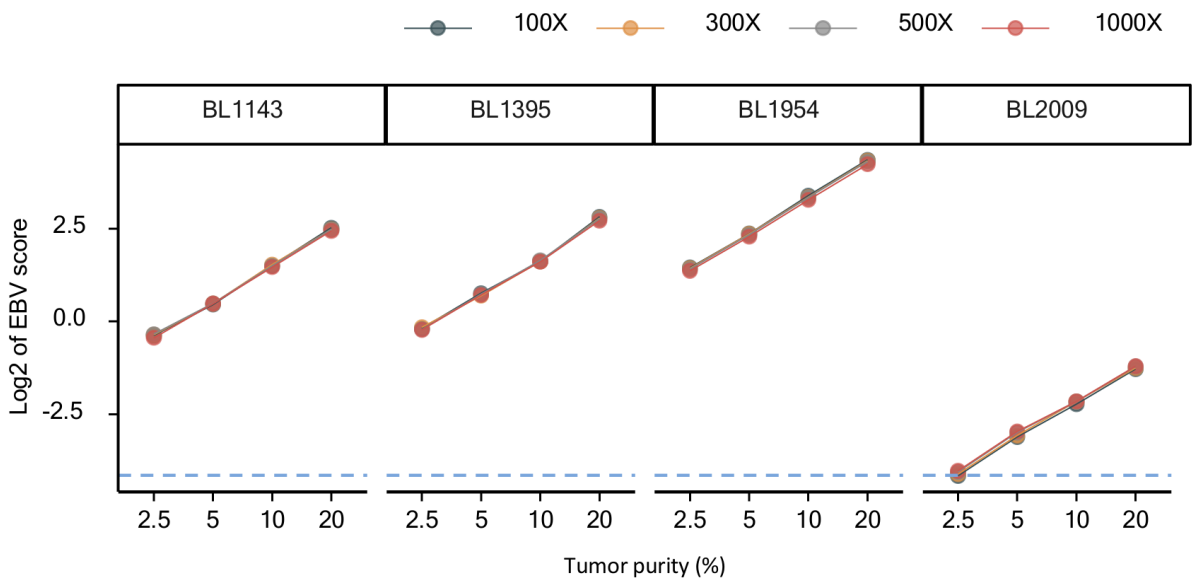

Figure 1 Establishment and validation of NGS-based EBV detection method. (A,) Normalized coverage of EBV genes in 124 GC tissue samples which EBV status was identified by EBER-ISH. (B,) NGS algorithm-developed EBV score of 124 GC tissue samples with EBV status identified by EBER-ISH. (C,) The linear correlation between NGS algorithm-developed EBV score and the EBV copy number determined by TaqMan probe based absolute quantitative PCR of BamHI W fragment in 20 tumor tissue samples from patients with GC. (D,) the ROC curve of EBV score for predicting EBV status in 76 GC tissue samples where EBV status was previously identified by EBER-ISH. The area under the ROC curve was 0.989 (95\% CI 0.968 to 1).(E,) Four EBV transformed cell lines were diluted to four concentrations $(2.5 \%, 5 \%, 10 \%$, and $20 \%)$ with blood white cells, and the normalized EBV score at each concentration under four sequencing depths $(100 \times, 300 \times, 500 \times$ and 1000x) were detected and calculated. AUC, area under the curve; EBV, Epstein-Barr virus; EBER, Epstein-Barr virus-encoded small RNA; GC, gastric carcinoma; ISH, in situ hybridization; NGS, next-generation sequencing; ROC, receiver operator characteristic. 
Table 1 Baseline characteristics of the patients with gastric carcinoma received ICB therapy

\begin{tabular}{|c|c|c|c|c|c|}
\hline Characteristics & $\begin{array}{l}\text { EBV+/pMMR } \\
(n=22)\end{array}$ & $\begin{array}{l}\text { EBV-/pMMR } \\
(n=44)\end{array}$ & $\begin{array}{l}\text { EBV-/dMMR } \\
(n=29)\end{array}$ & $\begin{array}{l}\text { P value: EBV+/pMMR } \\
\text { versus EBV-/pMMR }\end{array}$ & $\begin{array}{l}\text { P value: } E B V+/ p M M R \\
\text { versus } E B V-/ d M M R\end{array}$ \\
\hline $\begin{array}{l}\text { Age } \\
\text { Median (range) }\end{array}$ & $63.5(28-74)$ & $59.0(24-77)$ & $65.0(32-82)$ & 0.320 & 0.266 \\
\hline \multicolumn{6}{|c|}{ EBV status by EBER } \\
\hline Positive & 22 & 2 & 0 & 0.000 & 0.000 \\
\hline Negative & 0 & 36 & 23 & & \\
\hline Male & 18 & 31 & 19 & 0.384 & 0.225 \\
\hline Female & 4 & 13 & 10 & & \\
\hline \multicolumn{6}{|l|}{ Stage } \\
\hline III & 1 & 0 & 4 & 0.333 & 0.375 \\
\hline No & 9 & 1 & 6 & & \\
\hline \multicolumn{6}{|l|}{ ICB strategy } \\
\hline Mono-ICB & 8 & 43 & 25 & 0.000 & 0.000 \\
\hline Dual-ICB & 14 & 1 & 4 & & \\
\hline \multicolumn{6}{|l|}{ PD-L1 (CPS >1) } \\
\hline Positive & 15 & 19 & 13 & 0.115 & 1.000 \\
\hline Negative & 7 & 23 & 7 & & \\
\hline \multicolumn{6}{|l|}{ HER2 IHC staining } \\
\hline 0 & 16 & 26 & 18 & 0.088 & 0.906 \\
\hline
\end{tabular}

CPS, combined positive score; dMMR, mismatch repair deficient; Dual-ICB, combination anti-CTLA-4 plus anti-PD-1/L1 therapy; EBER, Epstein-Barr virus-encoded small RNA; EBV, Epstein-Barr virus; HER2, human epidermal growth factor receptor 2; ICB, immune checkpoint blockade; ICI, immune checkpoint inhibitor; IHC, immunohistochemistry; Mono-ICB, anti-PD-1/L1 monotherapy; PD-1, programmed death 1; PD-L1, programmed death-ligand 1; pMMR, mismatch repair proficient.

pMMR (online supplemental figure 5C,D). A multivariable Cox regression analysis also confirmed that EBV and dMMR had equal effectiveness in predicting PFS regardless of monotherapy or combination therapy (online supplemental figure $5 \mathrm{E}$ ).

\section{Identification of the predictive factors for ICB efficacy in patients with EBVaGC}

Based on published literature highlighting the role of EBV infection in promoting an inflamed tumor immune microenvironment (TME), ${ }^{25}$ the density of multiple lymphocyte subgroups and the proposed ICB biomarkerexpressed cells were determined in 22 EBVaGC. Three EBVaGC with no available tissues and two EBVaGC with poor-quality tissue samples were excluded from this analysis. The mIF assay revealed that only the density of cytotoxic T lymphocyte-associated antigen-4 (CTLA-4) ${ }^{+}$and $\mathrm{T}$ cell immunoglobulin-3 (TIM-3) ${ }^{+}$cells in the tumor was significantly higher in the ICB non-response group than in the response group, while no significant difference was observed in other cell subgroups between the ICB response and non-response group (figure 3A, online supplemental figure 7). The representative images of $\mathrm{mIF}$ staining were displayed in online supplemental figure 8 . In eight patients receiving programmed death-1/ligand 1 (PD-1/L1) monotherapy, CTLA-4 expression level in six patients who did not reach response was numerically higher than that in two patients with PR (data not shown). Additionally, in 14 patients receiving the combination of CTLA-4 and PD-1/L1 blockers, 10 reached PR, two showed SD, and two experienced PD. The patients receiving dual-ICB therapy had a better response than those who administrated mono-immunotherapy with a tendency toward statistical significance $(\mathrm{p}=0.074)$ (figure 3B). Accordingly, the dual-ICB-treated patients tended to have a better PFS than the patients undergoing mono-immunotherapy (online supplemental figure 9 ). Consistent results were observed in another three patients with EBVaGC with ICB as neoadjuvant therapy. 
A

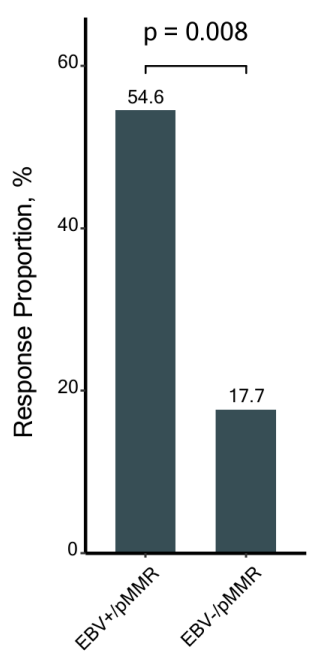

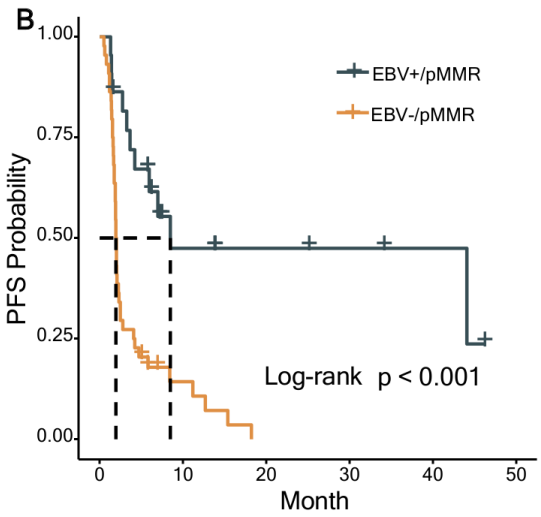

Number at risk

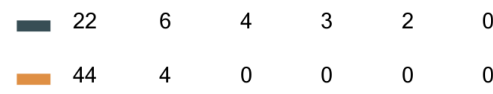

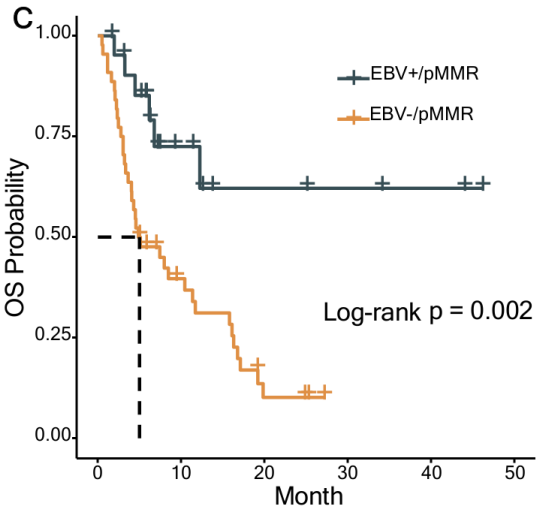

Number at risk

D

Uni-variate-cox analysis (PFS)

E

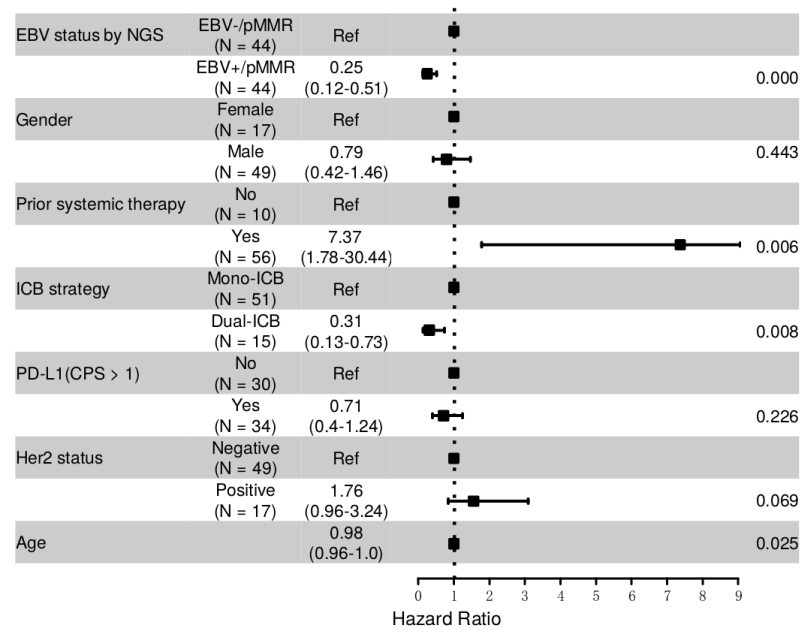

Multi-variate-cox analysis (PFS)

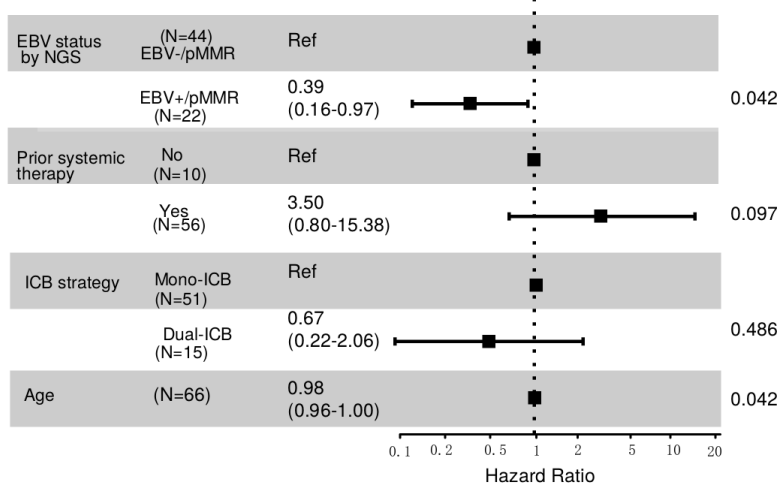

G

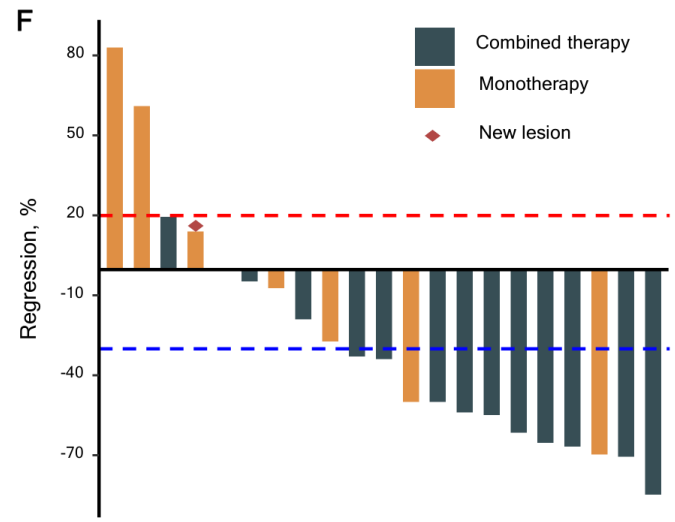

Figure 2 EBV infection as a predictive biomarker for patients with GC receiving ICB. A, the percentage of responders and non-responders in patients with EBV-/pMMR and EBV+/pMMR. Kaplan-Meier curve of PFS (B) and OS (C) in patients with pMMR receiving immunotherapy where EBV status was determined by NGS. Univariate (D) and multivariate (E) analysis of the variables associated with PFS of patients with pMMR treated with immunotherapy. Tumor regression from baseline $(F)$ and time to response and duration of response $(\mathrm{G})$ in 22 patients with EBVaGC receiving immunotherapy. CPS, combined positive score; CTLA-4, cytotoxic T lymphocyte-associated antigen-4; Dual-ICB, combination anti-CTLA-4 plus anti-PD-1/L1 therapy; EBV, Epstein-Barr virus; EBVaGC: Epstein-Barr virus-associated gastric carcinoma; GC, gastric cancer; ICB, immune checkpoint blockade; ICI, immune checkpoint inhibitor; Mono-ICB, anti-PD-1/L1 monotherapy; NGS, next-generation sequencing; PD, progressive disease; PD-1/L1, programmed death-1/ligand 1; PFS, progression-free survival; pMMR, mismatch repair proficient; $\mathrm{PR}$, partial response; SD, stable disease. 
A

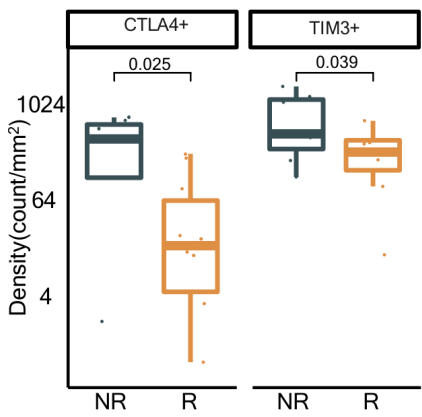

E

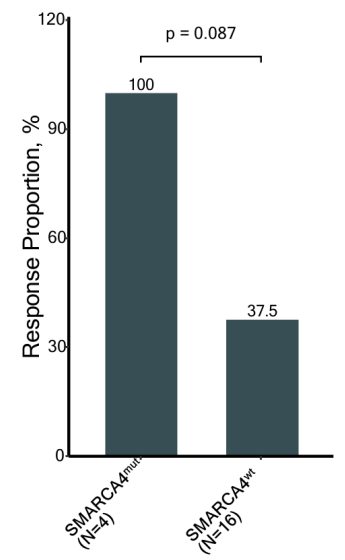

B

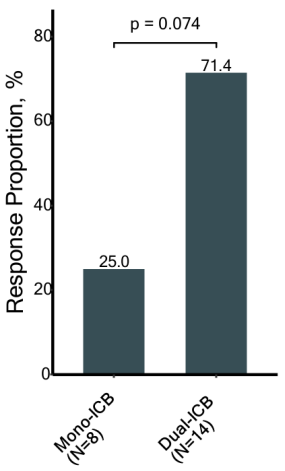

$\mathrm{F}$

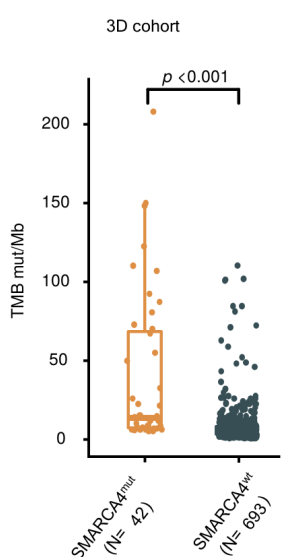

C

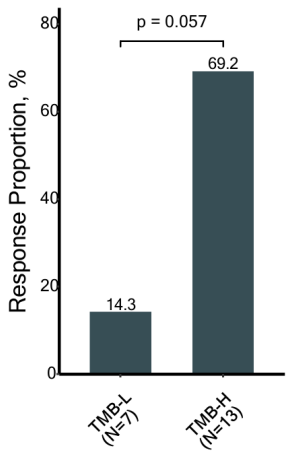

G

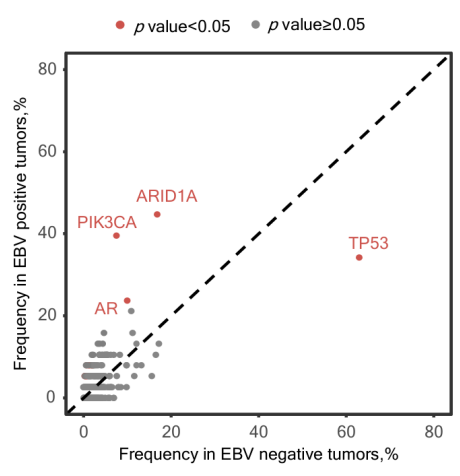

Figure 3 Biomarkers for patients with EBVaGC receiving ICB. Twenty-two patients with EBVaGC consisting of 12 ICB responders and 10 non-responders were analyzed to identify potential predictive biomarkers of ICB efficacy. (A), The density of CTLA $-4^{+}$and $\mathrm{TIM}^{+}$cells in tumor region from responders and non-responders. (B,) The percentage of responders and non-responders after administration of mono-immunotherapy or dual-immunotherapy. (C,) The percentage of responders and non-responders in TMB-low and TMB-high patients. (D,) Kaplan-Meier curve of PFS between TMB-low and TMB-high patients. $(E$,$) The percentage of responders and non-responders in the patients with or without SMARCA4 mutation. (F), The TMB level$ in $735 \mathrm{GC}$ cohort tumor tissues with or without SMARCA4 mutation from the 3DMed Biobank.(G,) The frequency variation of gene mutations between patients with EBVaGC and EBVnGC in $735 \mathrm{GC}$ cohort from 3DMed Biobank using a 733-gene panel. CTLA-4, cytotoxic T lymphocyte-associated antigen-4; Dual-ICB, combination anti-CTLA-4 plus anti-PD-1/L1 therapy; EBV, Epstein-Barr virus; EBVaGC, EBV-associated gastric cancer; EBVnGC, EBV-negative gastric cancer; ICB, immune checkpoint blockade; Mono-ICB, mono-immunotherapy; mut, mutation; NR, non-responder; PD-1/L1, programmed death-1/ligand 1; PFS, progression-free survival; R, Responder; SMARCA4, SWI/SNF related, matrix associated, actin dependent regulator of chromatin, subfamily A, member 4; Tim-3, T cell immunoglobulin-3; TMB, tumor mutation burden; TMB-H, high TMB; TMB-L, low TMB; WT, wild type.

The one with anti-PD-1 monotherapy did not reach clinical benefit, while two dual-ICB treated patients separately achieved pathological PR and pathological CR.

Since TMB also impacts the efficacy of immunotherapy in GC and its test has been recommended by the NCCN Guidelines, we also analyzed TMB level by NGS in 22 patients with EBVaGC, excluding two EBVaGC with poorquality tissue samples. TMB level was higher in the ICB response group than in the non-response group but failed to achieve a customary level of statistical significance $(\mathrm{p}=0.140)$ (data not shown). When 20 patients with EBVaGC were classified as TMB-high or TMB-low using the top quartile threshold (8.82 per $\mathrm{Mb})$ generated from
47 patients with EBVaGC with 24 in the training and 23 in the validation cohort, TMB-high patients had a significantly longer PFS (mPFS NR vs 3.2 months, $\mathrm{p}=0.024$ ) along with a trend toward better response (ORR 69.2\% vs $14.3 \%, p=0.057$ ) than TMB-low patients (figure 3C,D).

Next, the gene mutations were investigated. The frequently mutated genes were listed in online supplemental figure 10. SMARCA4 gene mutation occurred more commonly in the response group than the nonresponse group with a borderline level of statistical significance $(40 \%$ vs $0 \%, \mathrm{p}=0.087)$. All the patients with SMARCA4 mutation attained a PR following ICB and had a numerically higher PFS than wild-type SMARCA4 
patients (figure 3E, online supplemental figure 11). Additionally, the frequency of SMARCA4 mutation was analyzed and exhibited no significant difference between EBVaGC and EBVnGC in a 735 Chinese patients with GC cohort retrieved from the 3DMed Biobank (data not shown). These data suggested that SMARCA4 mutation might be a promising predictor of ICB efficacy in patients with EBVaGC.

Considering that patients with SMARCA4 mutation are reported to benefit more from ICB in non-small cell lung cancer via increasing $\mathrm{TMB},{ }^{26}$ the effects of SMARCA4 mutation on TMB were also analyzed. The patients with SMARCA4 alteration possessed a higher TMB level than patients with wild-type SMARCA4, which reached borderline significance in 20 patients with EBVaGC ( $\mathrm{p}=0.098$ ) and statistical significance in the Chinese GC cohort $(\mathrm{p}<0.001)$ (figure 3F).

Characterization of EBVaGC was performed via a 735-case Chinese GC cohort using our EBV algorithm along with the 733-gene panel. About 5.2\% (38/735) of the patients were identified as EBVaGC. The mutational profiles showed that mutations in ARID1A $(44.7 \%$ (17/38), $\mathrm{p}<0.001)$, PIK3CA (39.5\% (15/38), $\mathrm{p}<0.001)$, and $A R(23.7 \%(9 / 38), \mathrm{p}=0.029)$ were recurrent in EBVaGC, while alterations in TP53 $(63.0 \%$ (439/697), $\mathrm{p}<0.001$ ) were enriched in EBVnGC (figure 3G).

\section{DISCUSSION}

Herein we innovatively developed an NGS-based EBV detection strategy which allows for simultaneous characterization of other genomic features of patients with GC. Our EBV algorithm demonstrated a sensitivity of $95.7 \%$, a specificity of $100 \%$, and an overall accuracy of 98.7\% in reference to EBER-ISH in the validation cohort. Moreover, EBV infection predicted response in $54.5 \%$ of an ICB-treated cohort, which was as effective as dMMR in predicting favorable outcomes for patients with ICBtreated GC. TME analysis revealed that patients with EBVaGC with high CTLA-4 levels were less responsive to single-agent anti-PD-1/L1 therapy, and EBVaGC derived greater benefit from combination PD-1/L1 plus CTLA-4 blockade than anti-PD-1/L1 monotherapy. Further investigation identified that TMB and SMARCA4 mutation might be predictive biomarkers of ICB efficacy in patients with EBVaGC.

Ever since EBVaGC was recognized as a distinct molecular subtype, EBV has gained widespread attention as a potential biomarker to guide the personalized management of GC. ${ }^{27}$ Given the considerable heterogeneity of GC and the challenge with tissue availability, it is highly desirable to profile other biomarkers along with EBV in one single assay. ${ }^{28}{ }^{29}$ However, most previously reported NGS-based EBV detection involved sequencing of the entire EBV genome, and none of them incorporated EBV with cancer-related genes in the same panel, rending them less practical for clinical use. ${ }^{1516}$ In our EBV detection panel, the four EBV genes were analyzed with the cancer-related genes together, and therefore this method may be more cost-effective and feasible to be applied in clinical practice. Additionally, both algorithm development and validation were performed using clinical samples, ensuring the overall accuracy and reliability of the algorithm.

Advanced GC has a dismal prognosis with a 5-year survival rate of $<30 \%$ and a limited number of effective therapeutic options. ${ }^{30}$ Despite the approval of ICB for treating chemorefractory GC, response to monotherapy was reported in only $11.2 \%-12 \%$ of unselected patients. ${ }^{24} 3132$ Although Food and Drug Administration approved pembrolizumab for patients with PD-L1 positive chemorefractory GC, a combined positive score of $\geq 1$ was only associated with an ORR of $15.5 \%-22 \% .^{32} 33$ dMMR/ MSI-H represents an effective marker by predicting an ORR of $45.8 \%-85.7 \%$ among metastatic patients. ${ }^{24} 3234$ However, around $80 \%$ of patients with GC are classified as pMMR. EBVaGC is a particular subtype of GC, and EBV infection is considered to be a potential biomarker for the response to immunotherapy in GC. ${ }^{128-1024}$ In our cohort of 22 patients with advanced EBVaGC, a 54.5\% ORR and an mPFS lasting 8.47 months were achieved following ICB, which were significantly improved compared with the unselected patients with GC treated with mono-immunotherapy (11.2\% ORR and mPFS 1.61 months) and dual-ICB therapy (24\% ORR and mPFS 1.4 months). ${ }^{24} 3132$ Further analysis showed that EBV status was comparably effective as dMMR/MSI-H as an independent predictive factor for ICB efficacy in GC. Notably, two patients with EBV negativity by NGS but positivity by EBER-ISH had poor and comparable survival with patients with EBV-/pMMR. One had similar molecular characteristics with patients with EBVnGC, such as TP53 mutation (c.844C $>\mathrm{T}$ ), while the other had a deleterious BCOR mutation, which might account for the poor benefit from ICB for that BCOR deletion had been documented to perturb dendritic cell development. ${ }^{35}$ These phenomena might be reminiscent of the EBV status detected by NGS with high accuracy of ICB efficacy prediction and potential false positivity of EBV identification by EBER-ISH. Given the challenge of false-positive/false-negative EBER-ISH results, incorporating NGS-based methods may improve the diagnostic accuracy of EBV-related diseases and better inform follow-up treatment.

EBV infection has been reported to increase tumorinfiltrating lymphocytes and the expression of immune checkpoint molecules, thereby invoking clinical response to ICB. ${ }^{36}{ }^{37}$ We further investigated the TME difference between the ICB responders and non-responders in EBVaGC and found that the density of CTLA $-4^{+}$cells and TIM- $3^{+}$cells was significantly higher in the non-response group than in the response group. High CTLA-4 expression on helper $\mathrm{T}$ cells causes the immunosuppressive microenvironment. ${ }^{38}$ As expected, patients with EBVaGC with high CTLA-4 levels were less responsive to anti-PD-1/ L1 monotherapy, and EBVaGC derived more benefit from combination PD-1/L1 plus CTLA-4 blockade than 
anti-PD-1/L1 monotherapy. TIM-3, standing for T-cell immunoglobulin and mucin domain 3 , is a crucial immune checkpoint and negatively affects the immune system via complex biology. ${ }^{39} 40$ One recent study showed that the TIM- $3^{+}$cell infiltration was associated with an immunoevasive GC subtype with $\mathrm{CD}^{+} \mathrm{T}$ cell dysfunction and identified that TIM-3 might serve as a promising target for immunotherapy in GC. ${ }^{41}$ Another recent study reported that combining anti-PD-1 and anti-TIM-3 mAb had an additive effect on the cytotoxicity of cytotoxic $\mathrm{T}$ lymphocytes, suggesting the dual-ICB targeting for PD-1 and TIM-3 as a means of increasing response rates in GC. ${ }^{42}$ Based on these findings, triple blockade therapy targeting PD-1, CTLA-4, and TIM-3 might be a rational approach to benefit the patients with EBVaGC with the high density of CTLA- $4^{+}$and TIM- $3^{+}$cells.

Extensive analyses about genomic features were also conducted comparing ICB responders and nonresponders to find possible pretreatment biomarkers predictive of response or resistance. SMARCA4 is the most commonly mutated member of the chromatin remodeling SWI/SNF complex. Some evidence suggested that improved activity of ICB in SMARCA4-deficient cancers might be owing to the increased TMB and activated TME. $^{26} 43{ }^{44}$ Consistently, our findings from 22 EBVaGC and 735-case Chinese GC cohort showed that SMARCA4 mutation might be a positive predictor of ICB efficacy in EBVaGC, and the patients with SMARCA4 mutation attained PR, which might be owing to the increased TMB.

It has been documented that EBV-associated tumors have distinct molecular and TME characteristics, which may guide more targeted clinical treatment. ${ }^{45-47}$ Combining the EBV algorithm with the 733-gene panel, we identified EBV prevalence in a Chinese cohort of 735 patients with GC as well as the landscape of their molecular characteristics. EBVaGC accounted for $5.2 \%$ of the cohort, almost identical with the prevalence of $5.1 \%$ for EBV previously reported among Chinese patients with GC. ${ }^{3}$ Consistent with previous studies, ${ }^{45}{ }^{48-50}$ EBVaGC tumors were characterized with a high prevalence of ARID1A, PIK3CA, and AR mutations, all of which were associated with improved antitumor immunity or sensitivity to ICB in solid tumors, while EBVnGC had a higher frequency of mutations in TP53, whose mutations had been reported associated with poorer ICB efficacy in patients with pMMR GC, supporting that patients with EBVaGC had a greater likelihood of benefit from ICB than patients with EBVnGC. ${ }^{51-54}$

The relatively small sample size of the EBVaGC ICBtreatment cohort, although it was the largest to date, represents the main limitation of our study. This study included predictive biomarker analysis about ICB efficacy and identified that CTLA-4, TMB, and SMARCA4 mutation might be predictive biomarkers of ICB efficacy in EBVaGC. Due to the small sample sizes and ethnically homogeneous populations for these analyses, these results are challenging, and caution should be applied in extrapolating these results to patients of other ethnicities.
Prospective trials with larger sample sizes and different ethnic populations are warranted to confirm these findings.

In summary, our NGS-based EBV detection method is accurate and reliable and enables comprehensive molecular diagnosis of EBVaGC with specific implications for ICB efficacy prediction.

Acknowledgements We thank the staff Bei Zhang, Hui Chen, Shiqing Chen, Hao Chen, Jie Wang, Yunjie Song, Lei Wang, and Youbing Guo of the 3D Medicines for their valuable contributions in statistical analysis and critical revision of the manuscript for important intellectual content.

Contributors LS is responsible for the overall content as the guarantor. LS accepts full responsibility for the work and/or the conduct of the study, had access to the data, and controlled the decision to publish. LS and ZP conceived and designed this study and reviewed and corrected the manuscript. YB, TX, ZW, ST, XZ, FZ, and XW were involved in the data collection and interpretation. JC, ST, and XZ drafted the manuscript. FZ performed the statistical analysis. YB and TX revised the manuscript.

Funding This study was supported by the Fundamental Research Funds for the Central Universities/Peking University Clinical Medicine Plus X-Young Scholars Project (No. PKU2021LCXQ016), the third round of public welfare development and reform pilot projects of Beijing Municipal Medical Research Institutes (Beijing Medical Research Institute, 2019-1), and the Key Program of Beijing Natural Science Foundation (No. Z210015).

Competing interests ST, FZ, XZ, and JC are employees of 3D Medicines.

Patient consent for publication Not applicable.

Ethics approval The study was approved by the institutional review board of Beijing Cancer Hospital (Approval ID: 2019YJZ56). Participants gave informed consent to participate in the study before taking part.

Provenance and peer review Not commissioned; externally peer reviewed.

Data availability statement All data relevant to the study are included in the article or uploaded as supplementary information.

Supplemental material This content has been supplied by the author(s). It has not been vetted by BMJ Publishing Group Limited (BMJ) and may not have been peer-reviewed. Any opinions or recommendations discussed are solely those of the author(s) and are not endorsed by BMJ. BMJ disclaims all liability and responsibility arising from any reliance placed on the content. Where the content includes any translated material, BMJ does not warrant the accuracy and reliability of the translations (including but not limited to local regulations, clinical guidelines, terminology, drug names and drug dosages), and is not responsible for any error and/or omissions arising from translation and adaptation or otherwise.

Open access This is an open access article distributed in accordance with the Creative Commons Attribution Non Commercial (CC BY-NC 4.0) license, which permits others to distribute, remix, adapt, build upon this work non-commercially, and license their derivative works on different terms, provided the original work is properly cited, appropriate credit is given, any changes made indicated, and the use is non-commercial. See http://creativecommons.org/licenses/by-nc/4.0/.

ORCID iD

Lin Shen http://orcid.org/0000-0003-1134-2922

\section{REFERENCES}

1 Lee J-H, Kim S-H, Han S-H, et al. Clinicopathological and molecular characteristics of Epstein-Barr virus-associated gastric carcinoma: a meta-analysis. J Gastroenterol Hepatol 2009;24:354-65.

2 Cancer Genome Atlas Research Network. Comprehensive molecular characterization of gastric adenocarcinoma. Nature 2014;513:202-9.

3 Qiu M-Z, He C-Y, Lu S-X, et al. Prospective observation: clinical utility of plasma Epstein-Barr virus DNA load in ebv-associated gastric carcinoma patients. Int J Cancer 2020;146:272-80.

4 Chang MS, Kim DH, Roh JK, et al. Epstein-Barr virus-encoded BARF1 promotes proliferation of gastric carcinoma cells through regulation of NF-kB. J Virol 2013;87:10515-23.

5 van Beek J, zur Hausen A, Snel SN, et al. Morphological evidence of an activated cytotoxic T-cell infiltrate in EBV-positive gastric 
carcinoma preventing lymph node metastases. Am J Surg Pathol 2006;30:59-65.

6 Song H-J, Srivastava A, Lee J, et al. Host inflammatory response predicts survival of patients with Epstein-Barr virus-associated gastric carcinoma. Gastroenterology 2010;139:e82:84-92.

7 Liu X, Liu J, Qiu H, et al. Prognostic significance of Epstein-Barr virus infection in gastric cancer: a meta-analysis. BMC Cancer 2015;15:782.

8 Qiu M-Z, He C-Y, Yang D-J, et al. Observational cohort study of clinical outcome in Epstein-Barr virus associated gastric cancer patients. Ther Adv Med Oncol 2020;12:1758835920937434.

9 Kubota Y, Kawazoe A, Sasaki A, et al. The impact of molecular subtype on efficacy of chemotherapy and checkpoint inhibition in advanced gastric cancer. Clin Cancer Res 2020;26:3784-90.

10 Wang F, Wei XL, Wang FH, et al. Safety, efficacy and tumor mutational burden as a biomarker of overall survival benefit in chemo-refractory gastric cancer treated with toripalimab, a PD-1 antibody in phase lb/ll clinical trial NCT02915432. Ann Oncol 2019;30:1479-86.

11 Kim ST, Cristescu R, Bass AJ, et al. Comprehensive molecular characterization of clinical responses to PD-1 inhibition in metastatic gastric cancer. Nat Med 2018;24:1449-58.

12 Camargo MC, Bowlby R, Chu A, et al. Validation and calibration of next-generation sequencing to identify Epstein-Barr viruspositive gastric cancer in the cancer genome atlas. Gastric Cancer 2016;19:676-81.

13 Gulley ML. Molecular diagnosis of Epstein-Barr virus-related diseases. J Mol Diagn 2001;3:1-10.

14 (NCCN) NCCN. NCCN clinical practice Guildline in oncology. Gastric Cancer 2021

15 Liu P, Fang X, Feng Z, et al. Direct sequencing and characterization of a clinical isolate of Epstein-Barr virus from nasopharyngeal carcinoma tissue by using next-generation sequencing technology. $J$ Virol 2011:85:11291-9.

16 Chen J-N, Zhou L, Qiu X-M, et al. Determination and genomewide analysis of Epstein-Barr virus (EBV) sequences in ebvassociated gastric carcinoma from Guangdong, an endemic area of nasopharyngeal carcinoma. J Med Microbiol 2018;67:1614-27.

17 Gazdar AF, Kurvari V, Virmani A, et al. Characterization of paired tumor and non-tumor cell lines established from patients with breast cancer. Int J Cancer 1998;78:766-74.

18 Caputo JL. Biosafety procedures in cell culture. Journal of Tissue Culture Methods 1988;11:223-7.

19 Huang Q, Petros AM, Virgin HW, et al. Solution structure of the BHRF1 protein from Epstein-Barr virus, a homolog of human Bcl-2. J Mol Biol 2003;332:1123-30.

20 Lindquester GJ, Greer KA, Stewart JP, et al. Epstein-Barr virus IL-10 gene expression by a recombinant murine gammaherpesvirus in vivo enhances acute pathogenicity but does not affect latency or reactivation. Herpesviridae 2014;5:1.

21 Berenstein AJ, Lorenzetti MA, Preciado MV. Recombination rates along the entire Epstein Barr virus genome display a highly heterogeneous landscape. Infect Genet Evol 2018;65:96-103.

22 Farrell PJ. Epstein-Barr virus and cancer. Annu Rev Pathol 2019;14:29-53.

23 El-Sharkawy A, Al Zaidan L, Malki A. Epstein-Barr virus-associated malignancies: roles of viral oncoproteins in carcinogenesis. Front Oncol 2018;8:265

24 Janjigian YY, Bendell J, Calvo E, et al. CheckMate-032 study: efficacy and safety of nivolumab and nivolumab plus ipilimumab in patients with metastatic esophagogastric cancer. J Clin Oncol 2018;36:2836-44.

25 Gullo I, Oliveira P, Athelogou M, et al. New insights into the inflamed tumor immune microenvironment of gastric cancer with lymphoid stroma: from morphology and digital analysis to gene expression. Gastric Cancer 2019;22:77-90.

26 Schoenfeld AJ, Bandlamudi C, Lavery JA, et al. The Genomic Landscape of SMARCA4 Alterations and Associations with Outcomes in Patients with Lung Cancer. Clin Cancer Res 2020;26:5701-8

27 Röcken C. Molecular classification of gastric cancer. Expert Rev Mol Diagn 2017;17:293-301.

28 Robert ME, Lamps L, Lauwers GY, et al. Recommendations for the reporting of gastric carcinoma. Hum Pathol 2008;39:9.e1-9.e12.

29 Matsuoka T, Yashiro M. Biomarkers of gastric cancer: current topics and future perspective. World J Gastroenterol 2018;24:2818-32.

30 Chen W, Zheng R, Baade PD, et al. Cancer statistics in China, 2015. CA Cancer J Clin 2016;66:115-32.
31 Kang Y-K, Boku N, Satoh T, et al. Nivolumab in patients with advanced gastric or gastro-oesophageal junction cancer refractory to, or intolerant of, at least two previous chemotherapy regimens (ONO-4538-12, ATTRACTION-2): a randomised, double-blind, placebo-controlled, phase 3 trial. Lancet 2017;390:2461-71.

32 Fuchs CS, Doi T, Jang RW, et al. Safety and efficacy of pembrolizumab monotherapy in patients with previously treated advanced gastric and gastroesophageal junction cancer: phase 2 clinical KEYNOTE-059 trial. JAMA Oncol 2018;4:e180013.

33 Muro K, Chung HC, Shankaran V, et al. Pembrolizumab for patients with PD-L1-positive advanced gastric cancer (KEYNOTE-012): a multicentre, open-label, phase 1B trial. Lancet Oncol 2016;17:717-26.

34 Marabelle A, Le DT, Ascierto PA, et al. Efficacy of pembrolizumab in patients with Noncolorectal high microsatellite Instability/Mismatch repair-deficient cancer: results from the phase II KEYNOTE-158 study. J Clin Oncol 2020;38:1-10.

35 Tian L, Tomei S, Schreuder J, et al. Clonal multi-omics reveals BCOR as a negative regulator of emergency dendritic cell development. Immunity 2021;54:1338-51.

36 Derks S, de Klerk LK, Xu X, et al. Characterizing diversity in the tumor-immune microenvironment of distinct subclasses of gastroesophageal adenocarcinomas. Ann Oncol 2020;31:1011-20.

37 Panda A, Mehnert JM, Hirshfield KM, et al. Immune activation and benefit from Avelumab in EBV-positive gastric cancer. J Natl Cancer Inst 2018:110:316-20.

38 Leach DR, Krummel MF, Allison JP. Enhancement of antitumor immunity by CTLA-4 blockade. Science 1996;271:1734-6.

39 Solinas C, De Silva P, Bron D. Significance of Tim3 expression in cancer: from biology to the clinic. Paper presented at: Seminars in oncology, 2019.

40 Friedlaender A, Addeo A, Banna G. New emerging targets in cancer immunotherapy: the role of Tim3. ESMO Open 2019;4:e000497.

41 Chen K, Gu Y, Cao Y, et al. TIM3 ${ }^{+}$cells in gastric cancer: clinical correlates and association with immune context. $\mathrm{Br} J$ Cancer 2022;126:1-9.

42 Mimura K, Kua L-F, Xiao J-F, et al. Combined inhibition of PD-1/PDL1, Lag-3, and Tim-3 axes augments antitumor immunity in gastric cancer-T cell coculture models. Gastric Cancer 2021;24:611-23.

43 Takada K, Sugita S, Murase K, et al. Exceptionally rapid response to pembrolizumab in a SMARCA4-deficient thoracic sarcoma overexpressing PD-L1: a case report. Thorac Cancer 2019;10:2312-5.

44 Henon C, Blay J-Y, Massard C, et al. Long lasting major response to pembrolizumab in a thoracic malignant rhabdoid-like SMARCA4deficient tumor. Ann Oncol 2019;30:1401-3.

45 Hong S, Liu D, Luo S, et al. The genomic landscape of Epstein-Barr virus-associated pulmonary Lymphoepithelioma-like carcinoma. Nat Commun 2019;10:3108.

46 Huang Y-H, Zhang CZ-Y, Huang Q-S, et al. Clinicopathologic features, tumor immune microenvironment and genomic landscape of Epstein-Barr virus-associated intrahepatic cholangiocarcinoma. $J$ Hepatol 2021;74:838-49.

47 Derks S, Liao X, Chiaravalli AM, et al. Abundant PD-L1 expression in Epstein-Barr virus-infected gastric cancers. Oncotarget 2016;7:32925-32.

48 Wang K, Kan J, Yuen ST, et al. Exome sequencing identifies frequent mutation of ARID1A in molecular subtypes of gastric cancer. Nat Genet 2011;43:1219-23.

49 Böger C, Krüger S, Behrens HM, et al. Epstein-Barr virus-associated gastric cancer reveals intratumoral heterogeneity of PIK3CA mutations. Ann Oncol 2017;28:1005-14.

50 Zheng X, Wang J, Wei L, et al. Epstein-Barr virus microRNA miRBART5-3p inhibits p53 expression. J Virol 2018;92. doi:10.1128/ JVI.01022-18. [Epub ahead of print: 0112 2018].

51 Borcoman E, De La Rochere P, Richer W, et al. Inhibition of PI3K pathway increases immune infiltrate in muscle-invasive bladder cancer. Oncoimmunology 2019;8:e1581556.

52 Hu G, Tu W, Yang L, et al. ARID1A deficiency and immune checkpoint blockade therapy: from mechanisms to clinical application. Cancer Lett 2020;473:148-55.

53 Jiang G, Shi L, Zheng X, et al. Androgen receptor affects the response to immune checkpoint therapy by suppressing PD-L1 in hepatocellular carcinoma. Aging 2020;12:11466-84.

54 Wang JY, Xiu J, Baca Y, et al. Distinct genomic landscapes of gastroesophageal adenocarcinoma depending on PD-L1 expression identify mutations in Ras-MAPK pathway and TP53 as potential predictors of immunotherapy efficacy. Ann Oncol 2021;32:906-16. 